\title{
A regulatory function of long non-coding RNAs in red blood cell development
}

\author{
Klaudia Kulczyńska and Mirosława Siatecka ${ }^{\bowtie}$ \\ Department of Genetics, Institute of Experimental Biology, Faculty of Biology, Adam Mickiewicz University in Poznan, Poznań, Poland
}

In recent years it has been discovered that long noncoding RNAs are important regulators in many biological processes. In this review, we summarize the role of IncRNA in erythropoiesis. IncRNA are crucial for regulation of gene expression during both, proliferation and differentiation stages of red blood cell development. Many are regulated by erythroid-specific transcription factors and some are expressed in a developmental stage-specific manner. The majority of individually studied IncRNAs are involved in regulating the terminal maturation stages of red cell differentiation. Their regulatory function is accomplished by various mechanisms, including direct regulation in cis or trans by the IncRNA product or by the cis-localized presence of the IncRNA transcription itself. These add additional levels of regulation of gene expression during erythropoiesis.

Key words: long non-coding RNA, erythropoiesis, red blood cells

Received: 03 June, 2016; revised: 24 July, 2016; accepted: 15 August, 2016; available on-line: 17 November, 2016

\section{INTRODUCTION}

Erythropoiesis is a process characterized by commitment of pluripotent hematopoietic stem cells to the erythroid lineage. During mammalian development, erythropoiesis occurs successively in the yolk sac, the fetal liver and the bone marrow (Barker, 1968). Erythrocytes are short-lived, continuously replenished cells. Erythropoiesis comprises several developmental stages, during which hematopoietic stem cells are committed to progenitor and precursor cells with gradually restricted potential. In erythroid lineage development, a bipotential megakaryocytic-erythroid progenitor gives rise to a burst-forming unit-erythroid (BFU-E), which differentiates into a colony-forming unit-erythroid (CFU-E) and finally to erythroblasts. In hematopoietic tissue, these cells differentiate in the context of a specialized niche, the erythroblastic island, where erythroblasts are attached in concentric rings to one or more central macrophages. At this stage, cells decrease their size, nucleus is condensed and ultimately is expelled; cells become reticulocytes. The final stage of erythroid differentiation involves maturation of the reticulocytes into circulating, mature red blood cells (RBC). The reticulocytes dismantle their ribosomal machinery, expel organelles, and assume a biconcave discoid shape. (Manwani \& Bieker, 2008; An \& Mohandas, 2011; Palis, 2014).

To ensure a continuous and controlled production of red cells, this process has to be tightly regulated. Fundamental to the homeostasis of the hematopoietic system is the correct balance of progenitor cell proliferation versus lineage committed differentiation (Orkin \& Zon, 2008). Growth factors play a critical role in preventing apoptosis and in inducing proliferation, while successive combination of a complex network of transcription factors drives differentiation (Novershtern et al., 2011).

In recent years a new class of small, endogenous noncoding RNAs (ncRNAs) emerged as important regulators of gene expression at the post-transcriptional level. MicroRNAs are the best-known family of ncRNAs. These molecules are 19-23 nucleotides long and bind to specific sites within the 3'-UTR of regulated transcripts. MicroRNAs can decrease gene expression of various mRNAs by either inhibiting translation or directly causing degradation of the transcript. MicroRNAs demonstrate evolutionary conservation as well as tissue and developmental stage specific expression patterns (Wienholds \& Plasterk, 2005). In 2005, Lu and coworkers found for the first time that miRNAs expression changes during erythropoiesis (Lu et al., 2005; for reviews see: Lawrie, 2009; Listowski et al., 2013; Zhao et al., 2010).

This review focuses on long non-coding RNAs (lncRNAs) and their involvement in regulation of red blood cell development, particularly the late stages of adult erythropoiesis that comprise maturation of erythrocytes characterized by hemoglobinization, cell size reduction and extrusion of nucleus.

LncRNAs belong to a novel heterogeneous class of ncRNAs that includes thousands of different species identified by a high-throughput sequencing technologies that allow for the sequencing of the genome and transcriptome at an unprecedented depth (Paralkar \& Weiss, 2011; Alvarez-Dominguez et al., 2014; Paralkar et al., 2014). LncRNAs are longer than $200 \mathrm{nt}$, often polyadenylated and devoid of evident ORFs (Wilusz et al., 2009; Kung et al., 2013). LncRNAs can be classified based on genomic location relative to the well-established markers, such as protein-coding genes. Six classes can be distinguished (Table 1). The first class is located intergenically and does not overlap with any protein coding genes. The second class consists of lncRNAs situated within intronic regions (ilncRNA). In the third class, lncRNAs are transcribed from the antisense strand (alncRNA). The fourth class consists of enhancer lncRNAs (elncRNA), which are expressed from active enhancers. There is also a class named shlncRNA, where lncRNA hosts small RNA (sRNA) - microRNAs. A final class comprises pseudogene lncRNAs (plncRNA) (Alvarez-Dominguez et al., 2014; Rinn \& Chang, 2012). Unlike small ncRNAs,

e-mail: msiatecka@amu.edu.pl

Abbreviations: KLF1, Krüppel-like Factor 1; TAL1, T-Cell Acute Lymphocytic Leukemia 1; GATA1, GATA-binding factor 1; DLEU2, Deleted in Lymphocytic Leukemia 2; H3K4me3, histone 3 lysine 4 trimethylation; KIF2A, Kinesin Heavy Chain Member 2A; PIP5K, Phosphatidylinositol 4-Phosphate-5 kinase 
Table 1. Classification of IncRNAs based on their genomic location (taken from (Alvarez-Dominguez et al., 2014)) and examples of IncRNA involved in erythropoiesis.

\begin{tabular}{|c|c|}
\hline Classes of IncRNA mRNA $\square$ IncRNA $\square$ & IncRNAs - role in erythropoiesis \\
\hline Intergenic IncRNA (lincRNA) & $\begin{array}{l}\text { Linc RNA-EPS } \\
\text { - } \text { prosurvival, anti-apoptotic } \\
\text { IncRNA-aGT } \\
\text { - maintaining of an active chromatin structure } \\
\text { - } \text { upregulation of adult } \alpha^{\mathrm{D}} \text {-globin }\end{array}$ \\
\hline Intronic overlapping IncRNA (ilncRNA) & \\
\hline Antisense IncRNA (alncRNA) & $\begin{array}{l}\text { IncRNA Fas-antisense } 1 \\
\text { (IncRNA-Saf) } \\
\text { - conferring resistance to Fas-mediated cell death }\end{array}$ \\
\hline Enhancer IncRNA (elncRNA) & $\begin{array}{l}\text { AlncRNA-EC7 } \\
\text { - upregulation of SLC4A1 (Band 3 protein) } \\
\text { ElncRNA-EC3 } \\
\text { - upregulation of KIF2A }\end{array}$ \\
\hline sRNA-host IncRNA (shlncRNA) & $\begin{array}{c}\text { ShIncRNA-EC6/DLEU2 } \\
\text { (host to microRNAs 15a and 16-1) } \\
\text { - downregulation of SPRYD7/CLLD6 } \\
\text { - downregulation of Rac1-PIP5K } \\
\text { (promotion of enucleation) }\end{array}$ \\
\hline Pseudogene IncRNA (plncRNA) & \\
\hline
\end{tabular}

lncRNAs can fold into higher ordered structures to provide greater potential and versatility for target recognition (Batista \& Chang, 2013; Rinn \& Chang, 2012; Guttman \& Rinn, 2012). lncRNAs affect many biological processes, such as genomic imprinting, gene dosage compensation, gene expression, and nuclear organization. Several models have been proposed for the function of the lncRNAs: (1) as regulatory signals, (2) as a decoy system, (3) as guides of regulatory components to the genomic targets, and (4) as a ribonucleoprotein scaffold. The evidence indicates that lncRNAs exert their function mostly through modulation of chromatin-remodeling complexes (Wang \& Chang, 2011; Guttman \& Rinn, 2012; Rinn \& Chang, 2012; Arriaga-Canon et al., 2014).

Two major approaches have been applied to study involvement of lncRNA in erythropoiesis: 1) global analysis of lncRNA expression throughout RBC development using recent technologies, i.e., microarrays and next generation sequencing, 2) direct functional tests of various individual lncRNAs.

\section{GLOBAL ANALYSIS AND CHANGES IN InCRNA EXPRESSION DURING ERYTHROPOIESIS}

In the last couple of years several research groups performed a vast analysis of long non-coding RNAs involved in red blood cell development. Owing to newly developed technologies, such as high-throughput next generation sequencing and computational methods, they obtained comprehensive data sets from RNA-seq experiments. Diverse sources of erythroid cells were used for analyses covering different stages of red blood cell development, different source organs, and also various species. Researchers compared transcriptomes obtained from bipotential megakaryocytic-erythroid progenitors to lineage-committed megakaryocytes and erythroblasts using murine embryonic day 14.5 (E14.5) fetal liver and bone marrow cells. They identified 1109 potential lncRNA genes (including 683 transcribed in erythroblasts); around half of them were not annotated (Paralkar et al., 2014).

There were also studies of lncRNAs related to particular stages of erythroid development. Transcriptomes from BFU-E and CFU-E progenitors purified from mouse fetal liver along with differentiated TER119 positive erythroblasts were compared. As a result, 96 lncRNAs were identified that are differentially expressed during erythropoiesis (Alvarez-Dominguez et al., 2014), thus showing a dynamic regulation of $\operatorname{lncRNAs}$ expression during erythroid maturation. In addition, separate RNA-seq analyses were done for the TER119 positive cells comparing poly (A)+ and poly(A)- transcripts. In total, 9512 coding messenger RNAs (mRNA) genes and 655 lncRNA genes were identified (Alvarez-Dominguez et al., 2014). IncRNA included genes of all types of genomic location: intergenic, antisense, intronic, and enhancer loci, but also pseudogenes (Alvarez-Dominguez et al., 2014).

In general, these studies revealed that mRNAs were expressed at higher levels than lncRNAs, but lncRNAs were more developmental stage-restricted than coding mRNAs (Alvarez-Dominguez et al., 2014). ElncRNAEC1, lincRNA-EC9, and alncRNA-EC3 are expressed in erythroblasts but not in the closely related megakaryocyte or megakaryocyte-erythroid progenitors (AlvarezDominguez et al., 2014). 
Paralkar and coworkers compared lncRNA expression between fetal and adult erythropoiesis. More than $85 \%$ of fetal liver erythroid lncRNAs were detected in adult erythroblasts implying that most of mouse erythroid lncRNAs are expressed in both, fetal liver and adult bone marrow erythroblasts (Paralkar et al., 2014). However, Alvarez-Dominguez et al. showed that lncRNAs were expressed at different levels in mouse fetal and adult erythroblasts (Alvarez-Dominguez et al., 2014).

LncRNAs expressed in multiple species are less conserved in primary nucleotide sequence than coding genes (Paralkar et al., 2014). Paralkar et al. identified human orthologous regions for $95 \%$ of all transcribed mouse genes. Approximately $85 \%$ of coding genes expressed in mouse erythroblasts were also expressed in humans, but only $20 \%$ of erythroid lncRNA genes expressed in mouse erythroblasts were expressed in matched human samples. Similar results were obtained from an opposite analysis revealing that most mouse erythroid lncRNA genes are not transcribed in human erythroblasts. Only $15 \%$ of mouse lncRNAs are expressed in humans and vice versa, reflecting a dramatic species specificity (Paralkar et al., 2014). Most mouse erythro-megakaryocytic lncRNAs are transcribed from conventional gene promoters regulated by key hematopoietic transcription factors. Around $75 \%$ of erythro-megakaryocytic lncRNAs are transcribed from regions with promoter-like signatures and 25\% from enhancer-like signatures (Paralkar et al., 2014).

In erythroblasts, 60 of 96 differentially expressed lncRNAs are bound at their promoters by erythroid specific transcription factors such as GATA, TAL1, or KLF1 (Alvarez-Dominguez et al., 2014). Transcriptional binding for these factors coincides with DNase I hypersensitive sites, with RNA pol II binding and active epigenetic chromatin marks. As a consequence of regulation by cell-type specific transcription factors, lncRNA expression can be highly developmental stage-specific (Alvarez-Dominguez et al., 2014).

Sun and coworkers conducted RNA-seq studies comparing transcriptomes obtained from embryonic fetal liver tissues from wild-type and Klf1 knockout mice (Sun et al., 2012). Klf1 is a gene encoding an essential erythroid transcription factor (Miller \& Bieker, 1993; Siatecka \& Bieker, 2011). Klf1 knockout mice die from anemia by day E15, with severe defects in differentiation, hemoglobinization, enucleation, and membrane cytoskeleton organization of red blood cells (Nuez et al., 1995; Perkins et al., 1995). Transcriptome analyses identified 13 novel lncRNAs that showed significantly different expression between the wild-type and Klf1 knockout conditions (Sun et al., 2012). Ten lncRNAs were repressed versus three activated after Klf1 was knocked out. As described below, these may play functional roles in the development of erythroid cells.

\section{THE ROLE OF INDIVIDUAL InCRNAS IN ERYTHROID MATURATION}

Thus far, the majority of individually studied lncRNAs are involved in regulation of the terminal maturation stage in red cell development. The hallmarks of this stage of erythropoiesis are: expression of the TER119 marker, cell size reduction, progressive nuclear condensation and subsequent enucleation. Functions of lncRNAs were investigated through the knockdown procedure using shRNA.
LincRNA-EPS - lincRNA-EPS (erythroid prosurvival) was identified during erythroid differentiation of mouse fetal liver progenitors. LincRNA-EPS is located intergenically. It is $2531 \mathrm{nt}$ long, consists of 4 exons and 3 introns and has a 5'end cap structure and a 3' poly(A) tail. LincRNA-EPS is enriched in hematopoietic organs, such as spleen, bone marrow and fetal liver cells. It is strongly induced during the transition from erythroid precursors CFU-Es to hemoglobin synthesizing TER119+ ${ }^{+}$cells (Hu et al., 2011; Paralkar \& Weiss, 2011). The knockdown of LincRNA-EPS by shRNAs significantly reduced the accumulation of erythroid cells by inducing massive apoptosis, as revealed by cell cycle analysis. A significant fraction of analyzed cells was located in the sub-G1 population representing cells that underwent apoptosis and/or necrosis. Conversely, ectopic expression of LincRNA-EPS resulted in an increased number of cells localized in the $\mathrm{S}$ and $\mathrm{G} 2 / \mathrm{M}$ phases, indicating that overexpression of LincRNA-EPS protects erythroblasts from apoptosis. The time frame of LincRNA-EPS induction correlates well with the time window at which erythropoietin (Epo) exerts its biological function. This suggests that LincRNA-EPS's anti-apoptotic ability could contribute to cell survival mediated by Epo ( $\mathrm{Hu}$ et al., 2011).

$\mathrm{Hu}$ and coworkers found evidence indicating that Pycard, a signaling molecule that promotes cell death by activating caspases (Ohtsuka et al., 2004), is a target of LincRNA-EPS. Ectopic expression of LincRNA-EPS resulted in a dramatic repression of Pycard ( $\mathrm{Hu}$ et al., 2011). During normal erythropoiesis, the expression of Pycard is inversely correlated to LincRNA-EPS (Hu et al., 2011). Moreover, overexpression of Pycard inhibits proliferation of erythroid cells, promotes their apoptosis and interferes with their terminal differentiation and enucleation. The exact repression mechanism has not yet been identified (Hu et al., 2011; Paralkar \& Weiss, 2011).

Summarizing, the results obtained in the described research indicate that LincRNA-EPS modulates apoptosis at least in part through repressing Pycard expression. This pathway of regulation is required for the proper generation of mature red blood cells in response to various physiological and pathological stimuli.

ShlncRNA-EC6, also called DLEU2, is localized at chromosome 14. It belongs to lncRNA transcripts hosting microRNAs. ShlncRNA-EC6 hosts microRNAs 15a and 16-1. However, it has been suggested that the function of DLEU2 is independent of microRNA generation (Lerner et al., 2009; Klein et al., 2010; Alvarez-Dominguez et al., 2014). ShlncRNA-EC6 knockout or ectopic expression shows a stronger phenotype compared to miR-15a/16-1 knockout or misexpression. Shlnc-EC6 is broadly expressed. In erythroblasts, it is transcribed from a different specific promoter than in other cell types (Alvarez-Dominguez et al., 2014). Devoid of poly(A) tail shlncRNA-EC6/DLEU2 is predominantly induced during erythropoiesis. It promotes red blood cell maturation at least in part by cis-acting, lncRNA-directed control of expression of neighboring genes (Alvarez-Dominguez et al., 2014). Inhibition of DLEU2 caused up-regulation of SPRYD7/CLLD6, residing $45 \mathrm{~kb}$ away. No function is known for the SPRYD7 protein, although an RNA binding role has been proposed (Ponting et al., 1997).

Another study regarding shlnc-EC6 revealed its connection with the Rac1-PIP5K pathway. Knockdown of shlnc-EC6 in purified mouse fetal liver erythroid progenitors significantly blocked erythroid enucleation, which led to a significantly upregulated expression of Rac1 (Wang et al., 2015). Rac1 is a GTPase, and it has been 


\section{Regulation by target-specific IncRNA product}

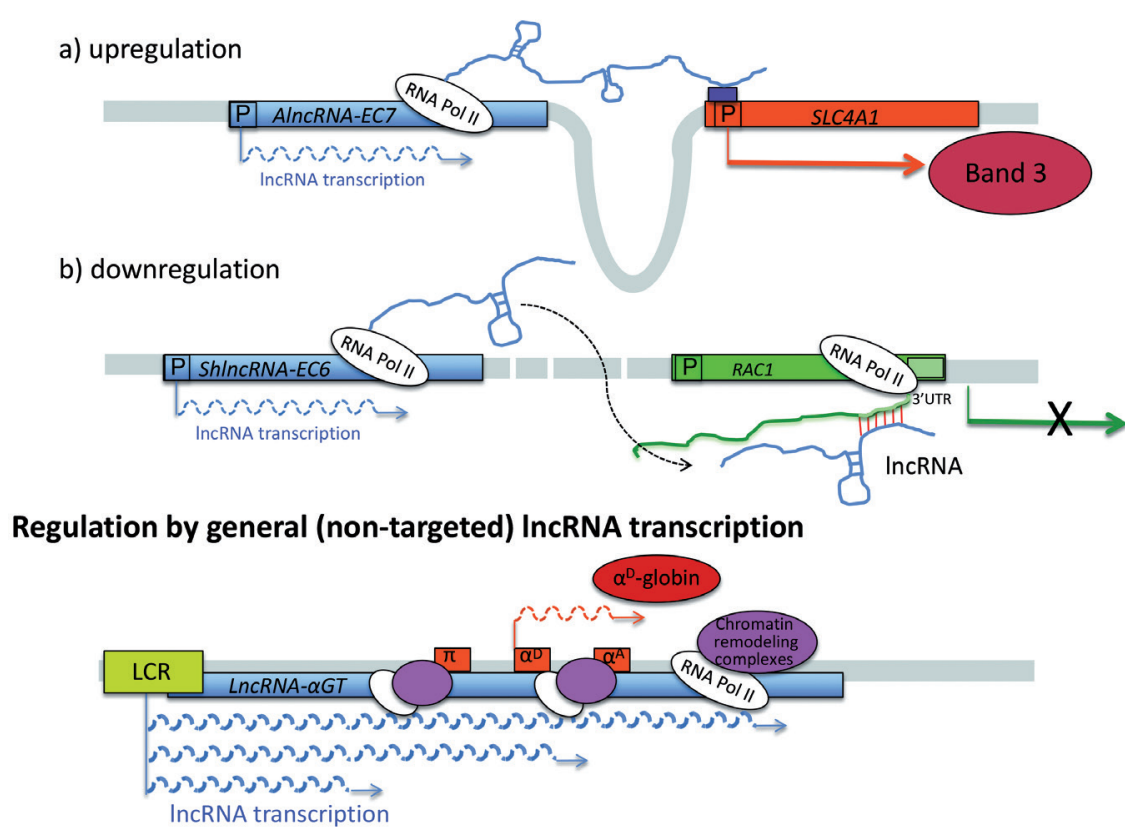

Figure 1. Modes of action of IncRNA involved in erythropoiesis.

Regulation by IncRNA product: a) upregulation of a cis target gene expression by induction of chromosomal looping or b) downregulation by posttranscriptional inhibition via specific binding of IncRNA in trans to sites within the $3^{\prime}$ UTR of the targeted mRNA. Regulation by IncRNA transcription process. The IncRNA gene overlaps with the targeted genes $(\pi, a \mathrm{D}, \mathrm{aA}-\mathrm{a}$-type globin genes; Gavrilov \& Razin, 2008) located on the same strand of DNA. Transcription of IncRNA recruits the epigenetic machinery to the chromatin which permits opening of its structure and allows for the transcription of the target genes (as delineated) along the same locus. P, promoter; LCR, locus control region.

reported that deregulation of Rac GTPase during the late stage of erythropoiesis blocks enucleation of cultured mouse fetal erythroblasts without affecting their proliferation or differentiation (Ji \& Lodish, 2010). Shlnc-EC6 negatively regulates Rac1 at the posttranscriptional level via specific binding to sites within the 3' UTR of Rac1 mRNA (Fig. 1) (Wang et al., 2015).

Consistently, overexpression of Rac1 and subsequent upregulation of its downstream component PIP5K strongly inhibited erythroid enucleation, which resembled the inhibitory effects of shlnc-EC6 knockdown (Villamizar et al., 2016). These results suggest that high expression of shlnc-EC6 at late-stage of red cell development helps erythroblasts to remove their nuclei through the Rac1-PIP5K pathway (Wang et al., 2015).

LncRNA Fas-antisense 1 (Fas-AS1 or Saf) is encoded on the antisense strand of the first intron of the human Fas receptor gene (also called APO-1 or CD95) on chromosome 10 (Yan, 2005). LncRNA Saf takes part in the maintenance of $\mathrm{RBC}$ production. Its promoter contains binding sites for the essential erythroid transcription factors GATA-1 and KLF1, as well as for $\mathrm{NF}-x \mathrm{~B}$. Experimental data support the significance of these sites for lncRNA Saf transcription. During the early stages of erythroblast expansion, NF- $x \mathrm{~B}$ signaling is involved in the repression of lncRNA Saf expression. Subsequently, at late stages of RBC maturation, expression of lncRNA Saf increases and it coincides with elevated expression of GATA-1 and KLF1, suggesting that lncRNA Saf could be regulated by these factors (Villamizar et al., 2016).

A cell culture model of human erythropoiesis revealed that induction of lncRNA Saf confers resistance to Fasmediated cell death. LncRNA Saf interacts with Fas premRNA and human splicing factor 45 (SPF45). It facili- tates splicing and production of a soluble Fas protein that protects cells against Fas-mediated apoptosis (Villamizar et al., 2016). Overexpression of lncRNA Saf in erythroblasts derived from CD34+ hematopoietic stem/ progenitor cells reduced surface levels of Fas receptor and protected cells from Fas-mediated apoptosis signals. LncRNA Saf expression may therefore provide a means to regulate cell death during erythroid maturation (Villamizar et al., 2016).

AlncRNA-EC7 is an enhancer transcript that regulates expression of SLCAA1 gene encoding Band 3 protein. Band 3 is a structural component of the erythrocyte cell membrane and the primary anion exchanger responsible for mediating the exchange of chloride $\left(\mathrm{Cl}^{-}\right)$with bicarbonate $\left(\mathrm{HCO}_{3}^{-}\right)$across plasma membrane (Alper, 2009). AlncRNA-EC7 is located $10 \mathrm{~kb}$ upstream from SLC4A1 gene locus and is involved in regulation of its expression by a cis mode of action. Experimental data suggested a model for looping of the alncRNA-EC7 enhancer to SLC4A1 gene locus with subsequent activation of Band 3 expression in erythroid cells (Fig. 1). Knockdown of alncRNA-EC7 was associated with an $80 \%$ decrease in Band 3 gene mRNA expression and severely impaired erythrocyte maturation, inhibiting cell size reduction and subsequent enucleation (Alvarez-Dominguez et al., 2014). As SLC4A1 gene is mutated in hereditary hemolytic anemias, this data predicts that alncRNA-EC7 is a novel disease-relevant locus (Sánchez-López et al., 2010).

ElncRNA-EC3 belongs to the enhancer class of $\ln$ cRNA and is transcribed from an erythroid-restricted enhancer co-bound at multiple sites by GATA1 and TAL1. Activation of elncRNA-EC3 in erythroblasts coincides with a more than two-fold up-regulation of its neighbor $\operatorname{KIF} 2 A$ gene that is located $40 \mathrm{~kb}$ away. Thus, eln- 
cRNA-EC3 may act in cis to enhance KIF2A expression in the erythroid lineage (Alvarez-Dominguez et al., 2014). $K I F 2 A$ gene encodes a kinesin family member $2 \mathrm{~A}$. It is a microtubule-associated motor protein that depolymerizes microtubules (Uehara et al., 2013). Depolymerization of microtubules might be necessary for nuclear polarization and that seems to connect it with enucelation. Presently, the role of this kinesin in erythropoiesis is unclear. Microtubules controlled by KIF2A are involved in normal mitosis progression (Homma et al., 2003),

lncRNA- $\alpha$ GT is an $\alpha$-globin transcript lncRNA studied in chicken. It is an approximately $23 \mathrm{~kb}$ long intergenic transcript synthesized along the $\alpha$-globin locus "in sense" with the globin genes (Gavrilov \& Razin, 2008). However, the level of transcription of $\ln$ cRNA- $\alpha$ GT is orders of magnitude lower as compared to transcription of $\alpha$-globin genes. LncRNA- $\alpha \mathrm{GT}$ is nuclear-restricted and its transcription occurs at late stages of chicken development, in terminally differentiated erythroid cells. The presence of lncRNA- $\alpha$ GT correlates with enrichment of $\mathrm{H} 3 \mathrm{~K} 4 \mathrm{me} 3$ over the adult $\alpha^{\mathrm{D}}$ gene promoter. Loss-of-function experiments have shown that IncRNA- $\alpha$ GT is required for full activation of the $\alpha^{\mathrm{D}}$ adult gene and maintenance of transcriptionally permissive chromatin. LncRNA- $\alpha$ GT is involved in switching from embryonic to adult $\alpha$-globin gene expression and later in maintaining of adult $\alpha$-globin gene expression by promoting an active, open chromatin structure (Arriaga-Canon et al., 2014).

Existence of similar intergenic lncRNAs had been detected for $\beta$-globin locus already over 15 years ago (Ashe et al., 1997; Bender et al., 2000; Gribnau et al., 2000; Ling et al., 2004, 2005). Transcription of these lncRNAs is initiated autonomously from the HS2 enhancer of the $\beta$-globin locus control region (LCR) (Ashe et al., 1997; Ling et al., 2005) and is independent of a cis-linked globin promoter. Generated transcripts are polyadenylated but differ in length. They delineate large regions of $15-30 \mathrm{~kb}$ surrounding active genes (Gribnau et al., 2000). No biological function has been correlated with these transcripts but because HS2 is unable to activate globin expression when a transcriptional terminator is placed between HS2 and the cislinked globin promoter, this phenomenon has been interpreted to be transcription- rather than transcriptdependent (Ling et al., 2004, 2005). The observed precise correlation between the extent of intergenic transcription and chromatin structure adjustments suggests a role of lncRNA- $\alpha$ GT in developmental remodeling of chromatin and establishing open chromatin structure marked by permissive histone modifications (Fig. 1) (Gribnau et al., 2000).

In conclusion, the lncRNAs detected for avian $\alpha$-globin locus, as well as intergenic lncRNA found for murine $\beta$-globin locus, have the same mode of action. In both cases, lncRNAs act through cis-regulatory mechanisms based on the transcription process itself that has the capacity to alter chromatin structure. This is crucial for the proper synthesis of specific globin products (Bender et al., 2000). During this process, transcriptional machinery containing RNA polymerase II, and also epigenetic modifiers such as SWI/SNF and histone acetyltransferases, cause an opening of the chromatin structure, allowing for access of other transcriptional factors. Consistent with this, developmental control of globin gene transcription is regulated primarily at the level of chromatin structure (Gribnau et al., 2000).

\section{CONCLUSIONS}

Many studies have recently identified and discussed the role of various lncRNAs that are involved in red blood cell development. LncRNAs add an additional level of regulation of gene expression. As opposed to microRNAs, their mechanism of action is more complex. Based on examples described above, modes of action of lncRNAs involved in erythropoiesis can be divided into two categories (Fig. 1): 1) regulation (up or down) by lncRNA product and 2) regulation by lncRNA transcription. These additional components that are important for erythroid regulation may also contribute to disease, but they may also provide new opportunities to use them as diagnostic markers and therapeutic targets for treatment of erythroid disorders.

\section{Acknowledgements}

The authors thank Dr. James Bieker from the Icahn School of Medicine at Mt.Sinai in New York, Dr. Barbara Imiołczyk from the Institute of Bioorganic Chemistry Polish Academy of Sciences and Dr. Karol Szkaradkiewicz from the Adam Mickiewicz University for helpful discussions and critical comments on this manuscript.

\section{Acknowledgements of financial support}

This work was supported by the OPUS grant 2013/09/B/NZ1/01879 of the Polish National Science Center, and the KNOW RNA Research Centre in Poznań (No. 01/KNOW2/2014).

\section{REFERENCES}

Alper SL (2009) Molecular physiology and genetics of $\mathrm{Na}^{+}-$independent SLC4 anion exchangers. J Exp Biol 212: 1672-1683. http://doi. org/10.1242/jeb.029454

Alvarez-Dominguez JR, Hu W, Yuan B, Shi J, Park SS, Gromatzky AA, Van Oudenaarden A, Lodish HF (2014) Global discovery of erythroid long noncoding RNAs reveals novel regulators of red cell maturation. Blood 123: 570-581. http://doi.org/10.1182/ blood-2013-10-530683

An X, Mohandas N (2011) Erythroblastic islands, terminal erythroid differentiation and reticulocyte maturation. Int I Hematol 93: 139_ 143. http://doi.org/10.1007/s12185-011-0779-x

Arriaga-Canon C, Fonseca-Guzmán Y, Valdes-Quezada C, Arzate-Mejía R, Guerrero G, Recillas-Targa F (2014) A long non-coding RNA promotes full activation of adult gene expression in the chicken $\alpha$-globin domain. Epigenetics 9: 173-181. http://doi.org/10.4161/ epi. 27030

Ashe HL, Monks J, Wijgerde M, Fraser P, Proudfoot NJ (1997) Intergenic transcription and transinduction of the human beta -globin locus. Genes Dev 11: 2494-2509. http://doi.org/10.1101/ $\operatorname{gad}$.11.19.2494

Barker JE (1968) Development of the mouse hematopoietic system. Dev Biol 18: 14-29. http://doi.org/10.1016/0012-1606(68)90020-1

Batista PJ, Chang HY (2013) Long noncoding RNAs: Cellular address codes in development and disease. Cell 152: 1298-1307. http://doi. org/10.1016/j.cell.2013.02.012

Bender M, Bulger M, Close J, Groudine M (2000) $\beta$-globin gene switching and DNase I sensitivity of the endogenous $\beta$-globin locus in mice do not require the locus control region. Mol Cell 5: 387393. http://doi.org/10.1016/S1097-2765(00)80433-5

Gavrilov AA, Razin SV (2008) Spatial configuration of the chicken -globin gene domain: immature and active chromatin hubs. Nucleic Acids Res 36: 4629-4640. http://doi.org/10.1093/nar/gkn429

Gribnau J, Diderich K, Pruzina S, Calzolari R, Fraser P (2000) Intergenic Transcription and developmental remodeling of chromatin subdomains in the human $\beta$-globin locus. Mol Cell 5: 377-386. http://doi.org/10.1016/S1097-2765(00)80432-3

Guttman M, Rinn JL (2012) Modular regulatory principles of large non-coding RNAs. Nature 482: 339-346. http://doi.org/10.1038/ nature 10887

Homma N, Takei Y, Tanaka Y, Nakata T, Terada S, Kikkawa M, Noda Y, Hirokawa N (2003) Kinesin superfamily protein 2A (KIF2A) functions in suppression of collateral branch extension. Cell 114: 229-239. http://doi.org/10.1016/S0092-8674(03)00522-1 
Hu W, Yuan B, Flygare J, Lodish HF (2011) Long noncoding RNAmediated anti-apoptotic activity in murine erythroid terminal differentiation. Genes Dev 25: 2573-2578. http://doi.org/10.1101/ gad.178780.111

Ji P, Lodish HF (2010) Rac GTPases play multiple roles in erythropoiesis. Haematologica 95: 2-4. http://doi.org/10.3324/haematol.2009.015511

Klein U, Lia M, Crespo M, Siegel R, Shen Q, Mo T, Ambesi-Impiombato A, Califano A, Migliazza A, Bhagat G, Dalla-Favera R (2010) The DLEU2/miR-15a/16-1 cluster controls b cell proliferation and its deletion leads to chronic lymphocytic leukemia. Cancer Cell 17: 28-40. http://doi.org/10.1016/j.ccr.2009.11.019

Kung JTY, Colognori D, Lee JT (2013) Long noncoding RNAs: past, present, and future. Genetics 193: 651-669. http://doi.org/10.1534/ genetics.112.146704

Lawrie CH (2009) microRNA expression in erythropoiesis and erythroid disorders. Brit J Haematol 150: 144-151. http://doi. org/10.1111/j.1365-2141.2009.07978.x

Lerner M, Harada M, Lovén J, Castro J, Davis Z, Oscier D, Henriksson M, Sangfelt O, Grandér D, Corcoran MM (2009) DLEU2, frequently deleted in malignancy, functions as a critical host gene of the cell cycle inhibitory microRNAs miR-15a and miR-16-1. Exp Cell Res 315: 2941-2952. http://doi.org/10.1016/j.yexcr.2009.07.001

Ling J, Ainol L, Zhang L, Yu X, Pi W, Tuan D (2004) HS2 Enhancer function is blocked by a transcriptional terminator inserted between the enhancer and the promoter. J Biol Chem 279: 51704-51713. http://doi.org/10.1074/jbc.M404039200

Ling J, Baibakov B, Pi W, Emerson BM, Tuan D (2005) The HS2 enhancer of the $\beta$-globin locus control region initiates synthesis of non-coding, polyadenylated RNAs independent of a cis-linked globin promoter. J Mol Biol 350: 883-896. http://doi.org/10.1016/j. jmb.2005.05.039

Listowski M, Heger E, Bogusławska D, Machnicka B, Kuliczkowski K, Leluk J, Sikorski A (2013) microRNAs: fine tuning of erythropoiesis. Cell Mol Biol Lett 18: 34-46. http://doi.org/10.2478/s11658-0120038-z

Lu J, Getz G, Miska E a, Alvarez-Saavedra E, Lamb J, Peck D, Sweet-Cordero A, Ebert BL, Mak RH, Ferrando A a, Downing JR, Jacks T, Horvitz HR, Golub TR (2005) MicroRNA expression profiles classify human cancers. Nature 435: 834-838. http://doi. org/10.1038/nature03702

Manwani D, Bieker JJ (2008) Chapter 2 The Erythroblastic Island. In Curr Topics Dev Biol pp 23-53. http://doi.org/10.1016/S00702153(07)00002-6

Miller IJ, Bieker JJ (1993) A novel, erythroid cell-specific murine transcription factor that binds to the CACCC element and is related to the Krüppel family of nuclear proteins. Mol Cell Biol 13: 2776-2786. http://doi.org/10.1128/MCB.13.5.2776

Novershtern N, Subramanian A, Lawton LN, Mak RH, Haining WN, McConkey ME, Habib N, Yosef N, Chang CY, Shay T, Frampton GM, Drake ACB, Leskov I, Nilsson B, Preffer F, Dombkowski D, Evans JW, Liefeld T, Smutko JS, Chen J, Friedman N, Young RA, Golub TR, Regev A, Ebert BL (2011) Densely interconnected transcriptional circuits control cell states in human hematopoiesis. Cell 144: 296-309. http://doi.org/10.1016/j.cell.2011.01.004

Nuez B, Michalovich D, Bygrave A, Ploemacher R, Grosveld F (1995) Defective haematopoiesis in fetal liver resulting from inactivation of the EKLF gene. Nature 375: 316-318. http://doi. org/10.1038/375316a0

Ohtsuka T, Ryu H, Minamishima Y a, Macip S, Sagara J, Nakayama KI, Aaronson S A, Lee SW (2004) ASC is a Bax adaptor and regulates the p53-Bax mitochondrial apoptosis pathway. Nature Cell Biol 6: 121-128. http://doi.org/10.1038/ncb1087

Orkin SH, Zon LI (2008) Hematopoiesis: an evolving paradigm for stem cell biology. Cell 132: 631-644. http://doi.org/10.1016/j. cell.2008.01.025

Palis J (2014) Primitive and definitive erythropoiesis in mammals. Front Physiol 5. http://dx.doi.org/10.3389/fphys.2014.00003
Paralkar VR, Mishra T, Luan J, Yao Y, Kossenkov A V., Anderson SM, Dunagin M, Pimkin M, Gore M, Sun D, Konuthula N, Raj A, An X, Mohandas N, Bodine DM, Hardison RC, Weiss MJ (2014) Lineage and species-specific long noncoding RNAs during erythromegakaryocytic development. Blood 123: 1927-1937. http://doi. org/10.1182/blood-2013-12-544494

Paralkar VR, Weiss MJ (2011a) A new "Linc" between noncoding RNAs and blood development. Genes Dev 25: 2555-2558. http:/9 doi.org/10.1101/gad.183020.111

Paralkar VR, Weiss MJ (2011b) A new 'Linc' between noncoding RNAs and blood development. Genes Dev 25: 2555-2558. http:// doi.org/10.1101/gad.183020.111

Perkins AC, Sharpe AH, Orkin SH (1995) Lethal $\beta$-thalassaemia in mice lacking the erythroid CACCC-transcription factor EKLF. Nature 375: 318-322. http://doi.org/10.1038/375318a0

Ponting C, Schultz J, Bork P (1997) SPRY domains in ryanodine receptors ( $\mathrm{Ca}^{2+}$-release channels). Trends Bioch Sci 22: 193-194. http:// doi.org/10.1016/S0968-0004(97)01049-9

Rinn JL, Chang HY (2012) Genome Regulation by Long Noncoding RNAs. Ann Rev Bioch 81: 145-166. http://doi.org/10.1146/annurevbiochem-051410-092902

Sánchez-López JY, Camacho-Torres AL, Ibarra B, Tintos JA, Perea FJ (2010) Analysis of the SLC4A1 gene in three Mexican patients with hereditary spherocytosis: report of a novel mutation. Genet Mol Biol 33: 9-11. http://doi.org/10.1590/S1415-47572009005000109

Siatecka M, Bieker JJ (2011) The multifunctional role of EKLF/ KLF1 during erythropoiesis. Blood 118: 2044-2054. http://doi. org/10.1182/blood-2011-03-331371

Sun L, Zhang Z, Bailey TL, Perkins AC, Tallack MR, Xu Z, Liu H (2012) Prediction of novel long non-coding RNAs based on RNASeq data of mouse Klf1 knockout study. BMC Bioinformatics 13: 331. http://doi.org/10.1186/1471-2105-13-331

Uehara R, Tsukada Y, Kamasaki T, Poser I, Yoda K, Gerlich DW, Goshima G (2013) Aurora B and Kif2A control microtubule length for assembly of a functional central spindle during anaphase. J Cell Biol 202: 623-636. http://doi.org/10.1083/jcb.201302123

Villamizar O, Chambers CB, Mo Y-Y, Torry DS, Hofstrand R, Riberdy JM, Persons DA, Wilber A (2016a) Fas-antisense long noncoding RNA is differentially expressed during maturation of human erythrocytes and confers resistance to Fas-mediated cell death. Blood Cells Mol Dis 58: 57-66. http://doi.org/10.1016/j.bcmd.2016.03.002

Villamizar O, Chambers CB, Mo Y-Y, Torry DS, Hofstrand R, Riberdy JM, Persons DA, Wilber A (2016b) Fas-antisense long noncoding RNA is differentially expressed during maturation of human erythrocytes and confers resistance to Fas-mediated cell death. Blood Cells Mol Dis 58: 57-66. http://doi.org/10.1016/j.bcmd.2016.03.002

Wang C, Wu X, Shen F, Li Y, Zhang Y, Yu D (2015) Shlnc-EC6 regulates murine erythroid enucleation by Rac1-PIP5K pathway. Development, Growth \& Differentiation 57: 466-473. http://doi.org/10.1111/ dgd. 12225

Wang KC, Chang HY (2011) Molecular mechanisms of long noncoding RNAs. Mol Cell 43: 904-914. http://doi.org/10.1016/j.molcel.2011.08.018

Wienholds E, Plasterk RHA (2005) MicroRNA function in animal development. FEBS Lett 579: 5911-5922. http://doi.org/10.1016/j. febslet.2005.07.070

Wilusz JE, Sunwoo H, Spector DL (2009) Long noncoding RNAs: functional surprises from the RNA world. Genes Dev 23: 1494-1504. http://doi.org/10.1101/gad.1800909

Yan M-D (2005) Identification and characterization of a novel gene Saf transcribed from the opposite strand of Fas. Human Mol Genet 14: 1465-1474. http://doi.org/10.1093/hmg/ddi156

Zhao G, Yu D, Weiss MJ (2010) MicroRNAs in erythropoiesis. Curr Opin Hematol 17: 1. http://doi.org/10.1097/ MOH.0b013e328337ba6c 